\title{
Erratum to: Epiretinal proliferation in lamellar macular holes and full-thickness macular holes: clinical and surgical findings
}

\author{
Tso-Ting Lai ${ }^{1}$ • San-Ni Chen ${ }^{2,3,4}$ • Chung-May Yang ${ }^{1,5}$
}

Published online: 25 September 2015

(C) Springer-Verlag Berlin Heidelberg 2015

\section{Erratum to: Graefes Arch Clin Exp Ophthalmol}

DOI 10.1007/s00417-015-3133-9

The original version of this article inadvertently contained a mistake.

Reason: Incorrect spelling of a cited name in the chapter "Discussion", first paragraph - i.e. "... In the LHEP group, color fundus showed that $57 \%$ had visible yellow pigment around the hole, which possibly indicated a xanthophyll pigment containing tissue around the hole margin, as had been mentioned by Peng et al. in their study [4]..."

Corrected version: "... In the LHEP group, color fundus showed that $57 \%$ had visible yellow pigment around the hole, which possibly indicated a xanthophyll pigment containing tissue around the hole margin, as had been mentioned by Pang et al. in their study [4]...."

The online version of the original article can be found at http://dx.doi.org/ 10.1007/s00417-015-3133-9.

Chung-May Yang

chungmay@ntu.edu.tw; chungmay100@gmail.com

1 Department of Ophthalmology, National Taiwan University Hospital, Taipei, Taiwan

2 Department of Ophthalmology, Changhua Christian Hospital, Changhua, Taiwan

3 Department of Medicine, Chung-Shan Medical University, Taichung, Taiwan

4 Department of Medicine, Kaohsiung Medical University, Kaohsiung, Taiwan

5 College of Medicine, National Taiwan University, Taipei, Taiwan 